\title{
STUDY OF AXISYMMETRIC STRESS-STRAIN STATE OF BILAYER CYLINDER
}

\author{
Volodymyr Bulgakovi ${ }^{1}$, Aivars Aboltins ${ }^{2}$, Oleksii Kutsenko ${ }^{3}$, Semjons Ivanovs ${ }^{2}$, Hristo Beloev ${ }^{4}$ \\ ${ }^{1}$ National University of Life and Environmental Sciences of Ukraine, Ukraine; \\ ${ }^{2}$ Latvia University of Life Sciences and Technologies, Latvia; \\ ${ }^{3}$ Taras Shevchenko National University of Kyiv, Ukraine; \\ 4"Angel Kanchev" University of Ruse, Bulgaria \\ semjons@apollo.lv
}

\begin{abstract}
The previously obtained solution of a nonstationary heat conduction problem for a two-piece rod, one end of which is in convective heat exchange with the environment, is generalized to the case of an inhomogeneous initial temperature field. The solution is presented as an eigenform expansion of the corresponding boundary problem and is expressed in terms of elementary functions. The obtained generalization makes it possible to propose an algorithm for solving a general problem with arbitrarily varying ambient temperature and heat exchange coefficient. The relations are presented, which allow to find axisymmetric stress fields in a two-layer cylinder by the calculated temperature field in a two-layer rod also in an analytical form. A comparative analysis has been made of the results obtained on the basis of the proposed analytical algorithm and the results of numerical calculations by the finite-element method. The aim of this comparison was to determine the influence of different simplifying assumptions used in constructing the algorithm on the accuracy of the results. These assumptions include: disregard of the cylinder curvature in the heat conductivity problem, disregard of the temperature dependence of material properties, change in the heat transfer coefficient. The comparison was performed using the example of a cylindrical body part under the thermal shock scenario. For these conditions, in general, the deviation of temperature and stresses calculated from the proposed approach from numerical results did not exceed $3 \%$. In this case, the main part of the error $-1.7 \%$ was introduced by a change in the dependence of physical properties on temperature. The second largest factor introduced into the calculation of the error was the failure to take into account the curvature of the cylinder when solving the problem of heat conduction. It corresponds to an error of about $1.1 \%$. Considering that the thermoshock scenario is boundary in terms of temperature change rate, it can be stated that the obtained analytical solution is sufficient for practical assessment of the axisymmetric stressstrain state of the corresponding equipment and pipelines.
\end{abstract}

Keywords: thermoshock, stresses, thermoelasticity, cylinder.

\section{Introduction}

Structural elements in the form of two-layer cylinders are widely used in the equipment of power engineering, transport, food industry, agriculture and other branches of economy. When investigating their strength, it is often necessary to calculate the temperature stress fields. These calculations are usually performed using the finite element method. In order to reasonably reduce the amount of calculations, it is important to have a simple way of estimating the criticality of the stress state occurring under various loading scenarios. After such an assessment, it is sufficient to perform finite element calculations only on the critical scenarios. The purpose of this work is to construct and validate an analytical solution of a nonstationary axisymmetric thermoelasticity problem for a two-layer cylinder, which inner surface is in a state of convective heat exchange with a fluid. The temperature of the fluid and the heat transfer coefficient vary with time. This solution should help in estimating the stress-strain state of the walls of cylindrical vessels and pipelines coated internally with corrosion-resistant cladding.

The analytical solution of the temperature problem for a homogeneous cylinder with a constant heat transfer coefficient has been known for a long time [1;2]. However, for a variable heat transfer coefficient, the search for an analytical solution capable of giving practically acceptable results even in the case of a homogeneous cylinder continues to this day [3;4].

In $[5 ; 6]$, a solution to the problem for a two-layer cylinder was given for the case where the coolant temperature changes by leaps or linearly in time, and the heat transfer coefficient is constant. In addition, it was assumed that the initial temperature field of the cylinder walls is homogeneous. To assess the stress state during the course of real scenarios, the assumption of constancy of the heat transfer coefficient cannot be acceptable, because the heat transfer coefficient varies within a wide range. So, for the equipment of the first circuit of nuclear reactors, these changes can be three orders of magnitude.

Note that under transient conditions the solution of the axisymmetric thermoelasticity problem allows us to approximate well the maximum values of the axial and circumferential stresses arising 
under a load that is non-uniform in the circumferential direction. In this case, the values of circumferential stresses are even somewhat more conservative [7].

Thus, the problem consists in determining the stress-strain state of a two-layer cylinder when the temperature of the external medium and its heat exchange coefficient with the inner wall of the cylinder change according to known laws. The specified problem implies a sequential solution of two problems: the problem of nonstationary thermal conductivity for a two-layer cylinder and the structural quasi-static problem of determining the temperature stresses using the known temperature distribution at each moment of time.

\section{Methods}

The radii of the vast majority of vessels and pipelines are much larger than their wall thicknesses. For example, for most cylindrical vessels, the ratio of the wall thickness of the cylindrical shell to its inner radius is close to 0.1 . This allows to locally neglect the wall curvature, when considering the axisymmetric heat conduction problem, i.e. to consider the problem not for a two-layer cylinder, but for a two-piece rectilinear rod of constant cross-section, the side surfaces of which are thermally insulated.

Thus, we will consider the process of heat propagation in a piecewise homogeneous rod with an isolated side surface consisting of two homogeneous parts " 1 " ( $\left.0<x<h_{1}\right)$ and " 2 " $\left(h_{1}<x<h_{2}+h_{1}=h\right.$, see Fig. 1). At the end of the rod $x=0$, corresponding to the inner surface of the cylinder, there is convective heat exchange with the external environment. The outer surface of the cylinder $(x=h)$ is considered to be thermally insulated.

To begin with, we assume that the heat transfer coefficient between the left end of the rod and the external environment $H$ is constant. We will also assume that the ambient temperature changes over time at a constant rate $V$. Denote by $T_{1}(x, t)$ and $T_{2}(x, t)$ functions-distributions of temperature on parts of the rod " 1 " and " 2 ". Then, at the appropriate choice of the zero temperature level, the condition at the left end of the rod can be written in the form:

$$
\left.\left(T_{1}-\frac{\lambda_{1}}{H} \frac{\partial T_{1}}{\partial x}\right)\right|_{x=0}=V t
$$

where $\lambda_{1}-$ coefficient of internal thermal conductivity of the material of the part " 1 "; $t$ - time.

The boundary conditions are supplemented by initial conditions of arbitrary form:

$$
\left.T_{k}\right|_{t=0}=T_{k 0}(x), k=1,2,
$$

where $T_{10}(x)$ and $T_{20}(x)$ are arbitrary functions, that set the initial temperature distribution on the left and right parts of the rod, respectively.

The solution of the formulated problem will be sought in the form of superposition:

$$
T_{k}(x, t)=T_{x k}(x, t)+T_{t k}(x, t), k=1,2,
$$

where $T_{x k}(x, t)$ - are the functions that provide satisfaction of the heterogeneous boundary condition (1);

$T_{t k}(x, t)$ - are the functions that provide satisfaction of heterogeneous initial conditions (2).

Problem solution with respect to functions $T_{x k}(x, t)$ was obtained in [5] by applying the Laplace transform over time [8]. It can be represented as:

$$
T_{x k}(x, t)=\frac{V}{\omega}\left[\psi_{k}(x, t)+2 \sum_{n=1}^{\infty} \frac{\Delta_{k}\left(\mu_{n}, x\right)}{\mu_{n}^{3} \Delta_{0}\left(\mu_{n}\right)} e^{-\mu_{n}^{2} \omega t}\right], k=1,2,
$$

where $\quad \Delta_{0}\left(\mu_{n}\right)=\delta_{1} \sin \mu_{n} \cos \beta \mu_{n}+\delta_{2} \cos \mu_{n} \sin \beta \mu_{n}+\delta_{3} \cos \mu_{n} \cos \beta \mu_{n}+\delta_{4} \sin \mu_{n} \sin \beta \mu_{n}$;

$\Delta_{1}\left(\mu_{n}, x\right)=\cos \beta \mu_{n} \cos \left(1-x / h_{1}\right) \mu_{n}-\alpha \sin \beta \mu_{n} \sin \left(1-x / h_{1}\right) \mu_{n} ;$

$\Delta_{2}\left(\mu_{n}, x\right)=\cos \left(1-\left(x-h_{1}\right) / h_{2}\right) \beta \mu_{n} ;$

$\psi_{1}(x, t)=\omega t+x^{2} h_{1}^{-2} / 2-(1+\alpha \beta)\left(\gamma+x / h_{1}\right), \omega=\left(a_{1} / h_{1}\right)^{2} ;$ 


$$
\begin{aligned}
& \psi_{2}(x, t)=\omega t-\beta^{2}\left(x-h_{1}\right)\left(1-\left(x-h_{1}\right) /\left(2 h_{2}\right)\right) / h_{2}-\alpha \beta(1+\gamma)-\gamma-1 / 2 ; \\
& \delta_{1}=1+\alpha \beta+\gamma, \delta_{2}=\alpha+\beta+\alpha \gamma, \delta_{3}=(1+\alpha \beta) \gamma \mu_{n}, \delta_{4}=-(\alpha+\beta) \gamma \mu_{n} ; \\
& \alpha=\frac{a_{1} \lambda_{2}}{a_{2} \lambda_{1}}, \beta=\frac{h_{2} a_{1}}{h_{1} a_{2}}, \gamma=\frac{\lambda_{1}}{h_{1} H}, a_{k}^{2}=\frac{\lambda_{k}}{\rho_{k} c_{k}} ; \\
& \lambda_{k} \text { - internal thermal conductivity coefficients; } \\
& \rho_{k} \text { - densities; } \\
& c_{k} \text { - heat capacities of the materials of the parts of the rod; } \\
& \mu_{n}, n=1,2,3 \ldots-\text { consecutive roots of a transcendental equation: } \\
& \cos \mu_{n} \cos \beta \mu_{n}-\alpha \sin \mu_{n} \sin \beta \mu_{n}-\gamma \mu_{n}\left(\sin \mu_{n} \cos \beta \mu_{n}+\alpha \cos \mu_{n} \sin \beta \mu_{n}\right)=0 .
\end{aligned}
$$

Relationships (4) and (5) completely define the functions $T_{x k}(x, t)$. The procedure for obtaining this solution is described in more detail in [5].

Turning to finding functions $T_{t k}(x, t)$, note that the series in (4) is the expansion in the system of eigenforms:

$$
X_{n}(x)=\left\{\begin{array}{cc}
\Delta_{1}\left(\mu_{n}, x\right), & 0<x<h_{1}, \\
\Delta_{2}\left(\mu_{n}, x\right), & h_{1}<x<h=h_{1}+h_{2} .
\end{array} .\right.
$$

The system of functions $X_{n}(x)$ as the solution of the eigenfunction problem is complete and orthogonal on the interval $[0 ; h]$, i.e.:

$$
\int_{0}^{h} X_{n}(x) X_{m}(x) d x=\left\{\begin{array}{cc}
0, & m \neq n \\
\left\|X_{n}\right\|, & m=n .
\end{array} .\right.
$$

This allows us to present the solution of the second problem for the functions $T_{t k}(x, t)$ in the form of a series of forms:

$$
T_{t}(x, t)=\left\{\begin{array}{ll}
T_{t 1}(x, t), & 0<x<h_{1}, \\
T_{t 2}(x, t), & h_{1}<x<h
\end{array}\right\}=\sum_{n=1}^{\infty} T_{n}(t) X_{n}(x) .
$$

We find the evolutionary multiplier $T_{n}(t)$ as a solution of Cauchy problems for first-order linear equations with constant coefficients, which we obtain by substituting (6) into the thermal conductivity equations and taking into account that the initial temperature distribution can also be decomposed into eigenforms:

$$
T_{0}(x)=\left\{\begin{array}{ll}
T_{10}(x), & 0<x<h_{1}, \\
T_{20}(x), & h_{1}<x<h
\end{array}\right\}=\sum_{n=1}^{\infty} a_{n} X_{n}(x) .
$$

where

$$
a_{n}=\left\|X_{n}\right\|^{-1} \int_{0}^{h} T_{0}(x) X_{n}(x) d x .
$$

Finally, the solution of the second problem can be written in the form:

$$
T_{t k}(x, t)=\sum_{n=1}^{\infty} a_{n} \Delta_{k}\left(\mu_{n}, x\right) e^{-\mu_{n}^{2} \omega t}, k=1,2 .
$$

The relations (3), (4) and (7) set the solution of the set problem. Based on this solution, it is possible to construct an algorithm for solving a more general problem for the case of arbitrary change in the heat transfer coefficient in time. For this purpose, the whole time interval of a transient process is divided into subintervals, in which the heat transfer coefficient changes little and can be considered constant. Its value is taken as averaged over the interval. Also, in each subinterval, the temperature change should be well approximated by a linear function. The formulated conditions can always be satisfied by choosing time subintervals small enough. After partitioning into subintervals, the general thermal conductivity problem is decomposed into a sequence of problems of the same type, the solution of which is given 
above. In this case, the initial temperature distribution in each interval, except for the first one, is obtained as the final distribution in the previous subinterval.

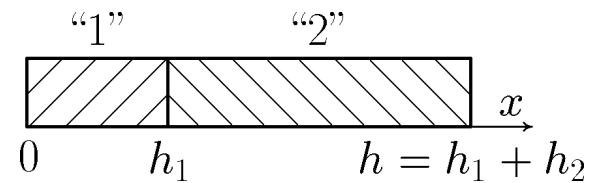

Fig. 1. Heat-transfer problem geometry

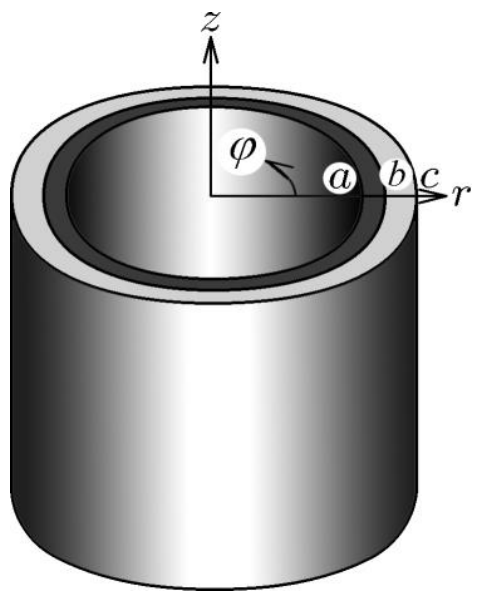

Fig. 2. Thermoelastic problem geometry

Temperature processes in the walls of vessels or pipelines develop much slower in time than the mechanical processes caused by the presence of intrinsic forces of inertia. Therefore, the structural problem can be regarded as quasi-static, in which time acts as a parameter, and a static problem of thermoelasticity is considered at each moment of time.

Thus, we consider the equilibrium of a cylinder consisting of two homogeneous elastic layers: the inner layer $a<r<b$, which parameters are denoted by the index " 1 ", and the outer layer $b<r<c$, which parameters are denoted by the index " 2 ". Here $r$ - the radial coordinate counted from the axis of the cylinder. To begin with, we will assume that no external mechanical loads are applied to the cylinder, and the external load corresponds to the non-uniform temperature field $T(x, t), a<r<c, t>0$. The temperature field for each moment of time is determined on the basis of the relations (3), (4) and (7). In doing so, we will assume that $r=x+a, h_{1}=b-a, h_{2}=c-b$. In practical calculations, the distributions of circumferential $\sigma_{\varphi}$ and longitudinal $\sigma_{z}$ stresses depending on the radial coordinate is of interest, first of all $r$.

Based on the equations in stresses, Hook's law and Cauchy relations for the components of the strain tensor, we can arrive at the ordinary differential equations with respect to the radial components of the displacement vector in each layer $u_{k}$. Their general solutions can be represented in the form [9]:

$$
u_{k}=\frac{1+v}{1-v} \alpha_{k} \frac{1}{r} \int_{r_{k}}^{r} \operatorname{Tr} d r+C_{2 k-1} r+\frac{C_{2 k}}{r}, k=1,2 .
$$

where $v$-Poisson's ratio, common for both materials,

$\alpha_{k}$ - coefficients of linear expansion of materials,

$C_{1-4}$ - arbitrary integration constants, $r_{1}=a, r_{2}=b$.

The following radial stress distributions in the layers correspond to the displacements (8):

$$
\sigma_{r k}=-\frac{\alpha_{k} E_{k}}{1-v} \frac{1}{r^{2}} \int_{r_{k}}^{r} \operatorname{Tr} d r+\frac{E_{k}}{1+v}\left(\frac{C_{2 k-1}}{1-2 v}-\frac{C_{2 k}}{r^{2}}\right), k=1,2 .
$$

where $E_{k}-$ Young's moduli of cylinder materials.

Subjecting (8) and (9) to the no-stress conditions on the side surfaces of the cylinder and to the continuity conditions for the displacement and stress fields at the interface transition, we find the values of the constants $C_{1}, C_{2}, C_{3}$ and $C_{4}$. Then the circumferential and longitudinal stresses in the cylinder layers can be represented as:

$$
\sigma_{\varphi 1}=\frac{\alpha_{1} E_{1}}{1-v} \frac{1}{r^{2}}\left[\frac{r^{2}+a^{2}}{b^{2}-a^{2}} \int_{a}^{b} T r d r+\int_{a}^{r} T r d r-T^{2}\right]-\frac{r^{2}+a^{2}}{b^{2}-a^{2}} \frac{b^{2}}{r^{2}} q,
$$




$$
\begin{gathered}
\sigma_{\varphi 2}=\frac{\alpha_{2} E_{2}}{1-v} \frac{1}{r^{2}}\left[\frac{r^{2}+b^{2}}{c^{2}-b^{2}} \int_{b}^{c} T r d r+\int_{b}^{r} T r d r-T r^{2}\right]+\frac{r^{2}+c^{2}}{c^{2}-b^{2}} \frac{b^{2}}{r^{2}} q, \\
\sigma_{z 1}=\frac{\alpha_{1} E_{1}}{1-v}\left[\frac{2}{b^{2}-a^{2}} \int_{a}^{b} T r d r-T\right]-\frac{Q}{b^{2}-a^{2}}, \sigma_{z 2}=\frac{\alpha_{2} E_{2}}{1-v}\left[\frac{2}{c^{2}-b^{2}} \int_{b}^{c} \operatorname{Tr} d r-T\right]+\frac{Q}{c^{2}-b^{2}},
\end{gathered}
$$

where

$$
q=\frac{\frac{2 \alpha_{1}}{b^{2}-a^{2}} \int_{a}^{b} \operatorname{Tr} d r-\frac{2 \alpha_{2}}{c^{2}-b^{2}} \int_{b}^{c} \operatorname{Tr} d r}{\frac{a^{2}+(1-2 v) b^{2}}{\left(b^{2}-a^{2}\right) E_{1}}+\frac{c^{2}+(1-2 v) b^{2}}{\left(c^{2}-b^{2}\right) E_{2}}}, Q=q\left[2 v b^{2}+\frac{\frac{a^{2}+(1-2 v) b^{2}}{\left(b^{2}-a^{2}\right) E_{1}}+\frac{c^{2}+(1-2 v) b^{2}}{\left(c^{2}-b^{2}\right) E_{2}}}{\frac{1}{\left(b^{2}-a^{2}\right) E_{1}}+\frac{1}{\left(c^{2}-b^{2}\right) E_{2}}}\right] .
$$

Since the liquid filling the vessels and pipelines is usually under pressure, the solution (10) must be supplemented by the solution of the problem for a bilayer cylinder, on the inner surface of which the pressure is applied $p$. It is constructed in a similar way and can be represented as:

$$
\begin{gathered}
\sigma_{\varphi 1}=\frac{p a^{2}-q b^{2}}{b^{2}-a^{2}}+\frac{(p-q) a^{2} b^{2}}{\left(b^{2}-a^{2}\right) r^{2}}, \sigma_{\varphi 2}=\frac{q b^{2}}{c^{2}-b^{2}}\left(1+\frac{c^{2}}{r^{2}}\right), \\
\sigma_{z 1}=2 v \frac{p a^{2}-q b^{2}}{b^{2}-a^{2}}+E_{1} Q, \sigma_{z 2}=2 v \frac{q b^{2}}{c^{2}-b^{2}}+E_{2} Q
\end{gathered}
$$

where

$$
q=\frac{\frac{2(1-v) a^{2}}{\left(b^{2}-a^{2}\right) E_{1}} p}{\frac{a^{2}+(1-2 v) b^{2}}{\left(b^{2}-a^{2}\right) E_{1}}+\frac{c^{2}+(1-2 v) b^{2}}{\left(c^{2}-b^{2}\right) E_{2}}}, Q=\frac{(1-2 v) a^{2} p}{\left(b^{2}-a^{2}\right) E_{1}+\left(c^{2}-b^{2}\right) E_{2}} .
$$

Relations (10) and (11) are given with some inaccuracies in [10]. They specify the solution of the axisymmetric thermoelasticity problem for a bilayer cylinder when the internal pressure and temperature distribution over the thickness of its wall are known.

\section{Results and discussion}

As an example, on which the possibility of applying the obtained solution to practical calculations was investigated, we considered the model scenario of a thermal shock of a cylindrical body given in [11]. Dependences of its thermal-hydraulic parameters on time (coolant temperature $T$, heat transfer coefficient $H$ and internal pressure $p$ ) are shown in Fig. 3. This scenario assumes a rapid temperature drop of about $200{ }^{\circ} \mathrm{C}$ during the first stage of loading. The points on the graphs correspond to key points in time. It is assumed that at the time intervals between the key moments all thermal-hydraulic parameters change linearly in time.

During the calculations, the geometric parameters of the cylinder were set equal to the geometric parameters of the cylindrical body shell: inner radius $a=2068 \mathrm{~mm}$, cladding thickness $h_{1}=7 \mathrm{~mm}$, base metal thickness $h_{2}=192.5 \mathrm{~mm}$. The thermal and elastic properties of the materials were chosen similar to those of [11].

The factors that affect the accuracy of the analytical solution compared to the numerical results can include:

- not taking into account the cylinder curvature in the analytical solution of the heat conduction problem;

- failure to take into account the dependence of material properties on temperature;

- jump change in the value of the heat transfer coefficient.

Using the results of [11] and additional calculations with the finite element package CalculiX [12], the contribution of each of these factors to the error of the final result was evaluated. The need for 
additional finite-element calculations was dictated by the limited data given in [11]. The analytical calculations were given for two degrees of discretization of the heat transfer coefficient: with its averaging at intervals between key moments of time and with averaging at each step. Let us denote the first analytical calculation as A1, and the second analytical calculation as A2. We note at once the fact that the numerical calculations, which do not take into account the continuous change in the heat transfer coefficient, the curvature of the cylinder and the dependence of material properties on temperature, coincided with the analytical solution with great accuracy (deviations are much less than $1 \%$ ), which confirms the correctness of the finding. In addition, three more numerical calculations were performed. Let us refer to these calculations as $\mathrm{C} 1, \mathrm{C} 2$, and $\mathrm{C} 3$. Calculation $\mathrm{C} 1$ took into account only the continuous change in the heat transfer coefficient, but did not take into account the curvature of the cylinder and the temperature dependence of the material properties. The calculation of $\mathrm{C} 2$, in addition to the continuous change in the heat transfer coefficient, also took into account the curvature of the cylinder, and the calculation of $\mathrm{C} 3$ took into account all three factors. In fact, the calculation of $\mathrm{C} 3$ repeated the calculation of the temperature field performed in [11], and their results coincide with great accuracy. Therefore, we will identify these calculations, and we will consider the calculation of $\mathrm{C} 3$ as a reference and use its results where the results given in [11] are missing.

Figure 4 compares the temperature evolution over time at six points: 1 - point on the inside surface of the cylinder, 2 - point on the interface, 3 - point $1 / 8$ of the base metal thickness from the interface, 4 - point $1 / 4$ of the base metal thickness from the interface, 5 - point in the middle of the base metal and 6 - point on the outside surface of the cylinder. The temperature values at points 2 and 3 for calculating $\mathrm{C} 3$ were taken from [11]. It can be seen that all the graphs break up into six consecutive families of curves depending on the calculated point. The lower family corresponds to point 1 , and the upper one to point 6 . Within each family, the curves are practically indistinguishable within the accuracy of the graphs, which indicates a good coincidence of the calculation results. To quantify the accuracy of the calculations, Table 1 shows the relative errors of the temperature calculations (second column) related to the $\mathrm{C} 3$ calculation results. The temperature difference at the beginning and at the end of the process was chosen as the normalizing temperature, i.e. $260{ }^{\circ} \mathrm{C}$.

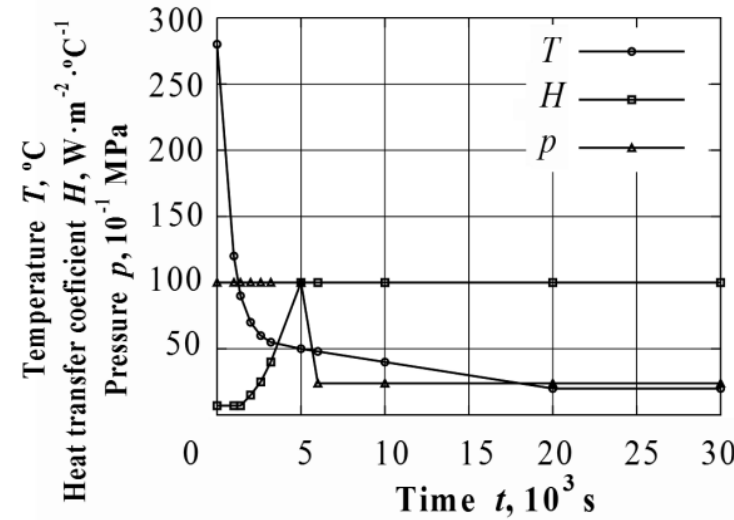

Fig. 3. Thermalhydraulics parameters of scenario

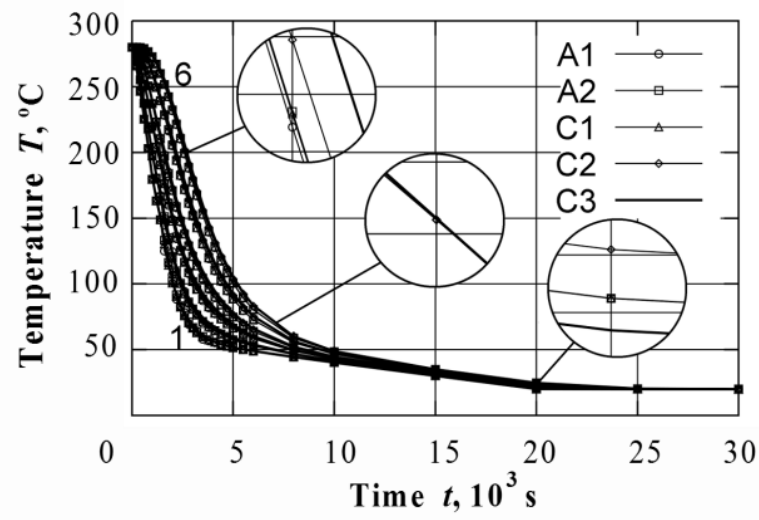

Fig. 4. Temperature evolution at six points obtained from different calculations

The calculations were performed on a uniform grid of points according to the depth of layers with a step of $0.35 \mathrm{~mm}$ in the cladding and $0.48 \mathrm{~mm}$ in the base metal of the CR.

Relative error of calculations

Table 1

\begin{tabular}{|c|c|c|c|}
\hline \multirow{2}{*}{ Calculation } & \multicolumn{3}{|c|}{ Error, \% } \\
\cline { 2 - 4 } & $T$ & $\sigma_{\varphi}$ & $\sigma_{z}$ \\
\hline $\mathrm{A} 1$ & 3.3 & 5.1 & 5.2 \\
\hline $\mathrm{A} 2$ & 2.8 & 2.6 & 2.8 \\
\hline $\mathrm{C} 1$ & 2.8 & 2.9 & 3.2 \\
\hline $\mathrm{C} 2$ & 1.7 & 1.9 & 2.0 \\
\hline
\end{tabular}


Several important conclusions can be drawn from the data in the second column of Table 1. First of all, note that all calculations provide acceptable practical accuracy. The jump change in the heat transfer coefficient weakly affects the accuracy of the results, because the difference in the error between scenarios A1 and A2 was only $0.5 \%$, and between scenarios A2 and $\mathrm{C} 1$ is negligibly small at all. Thus, we can state the fact that the piecewise constant approximation of the heat transfer coefficient is sufficient for practical calculations. The factor of not taking into account the curvature of the cylinder affects the accuracy of the results more significantly (the difference in the error between scenarios $\mathrm{C} 1$ and $\mathrm{C} 2$ is $1.1 \%$ ). Obviously, the corresponding error will grow as the ratio of the wall thickness to the cylinder radius increases (recall that in our case this ratio is close to 0.1 ). For relatively thick cylinders, the thermal conductivity problem should already be formulated as axisymmetric in polar coordinates, which will lead to replacement of trigonometric functions by cylindrical ones in the solution. Since the error from averaging of thermal properties is $1.7 \%$, which is higher than the error caused by not taking curvature into account, for relative wall thicknesses as in CR and smaller the transition to the axisymmetric formulation is unjustified in carrying out practical calculations. The constructed analytical solution corresponds to the problem in the linear formulation, so the procedure of averaging the thermophysical properties of materials cannot be bypassed in its framework and it is difficult to reduce the corresponding error in the results.

The main purpose of strength calculations of cylindrical bodies is to determine circumferential and axial stresses in them. To determine them, we performed calculations using the temperature fields obtained earlier in the calculations A1, A2, C1, C2 and C3. The plastic properties of the materials were not taken into account, i.e. the stresses were calculated for linearly elastic materials. Formulas (10), (11) were used to obtain an analytical solution. The temperature of zero voltages was considered to be $290^{\circ} \mathrm{C}$. Figures 5 and 6 show plots of the above stresses vs. time in seven points: 1 - point on the inside surface of the cylinder, 2 - point near the interface on the cladding side, $3-7$ - points in the base metal, evenly spaced along its thickness, beginning with point 3 near the interface and ending with point 7 on the outside surface of the cylinder.

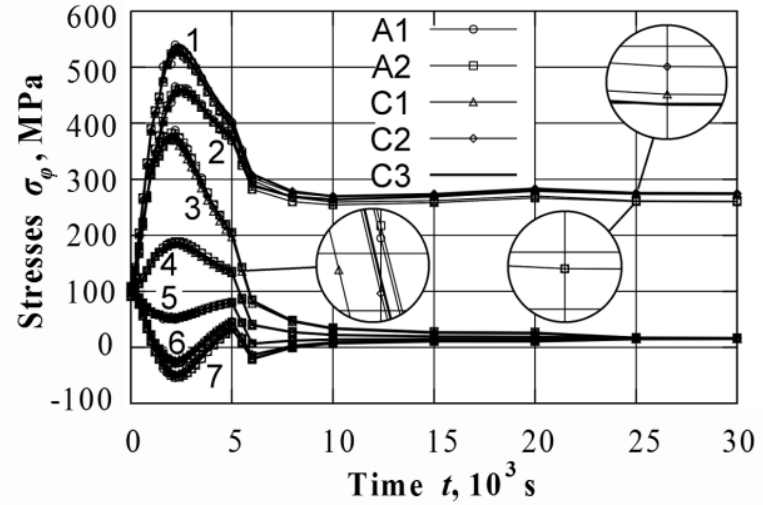

Fig. 5. Circumferential stresses at seven points obtained from different calculations

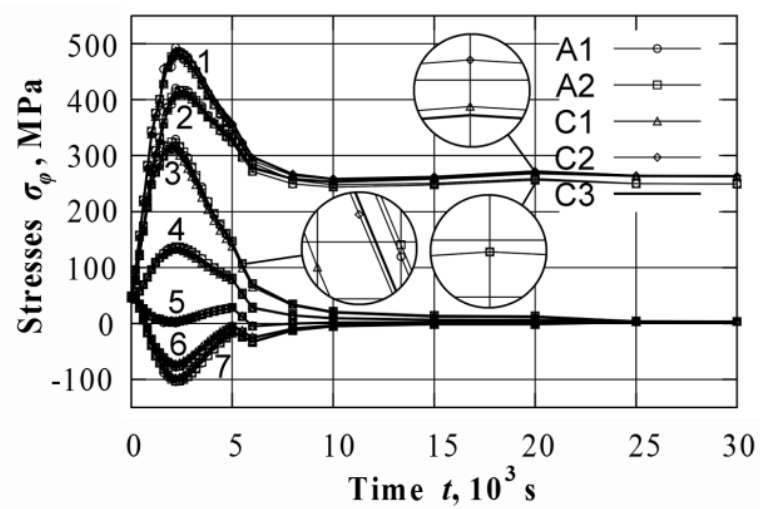

Fig. 6. Longitudinal stresses at seven points obtained from different calculations

As with the temperature plots, all curves are distributed into families corresponding to one point or another. The only exception is the points in the cladding, at which the curves corresponding to the analytical and numerical solutions were separated at the stage of temperature equalization in the cylinder wall. This is explained by the difference in the values of the averaged characteristics and the values corresponding to low temperatures, as well as by the much smaller thickness of the cladding layer in relation to the thickness of the base metal layer.

In order to quantify the influence of factors, we calculated the relative errors of the results of calculations of circumferential $\sigma_{\varphi}$ and axial $\sigma_{z}$ voltages A1, A2, C1 and C2 with respect to the calculation of $\mathrm{C} 3$. They are given in the third and fourth columns of Table 1. The maximum value of the corresponding stresses for the $\mathrm{C} 3$ calculation was taken as the normalizing value to which the deviation referred. The conclusions that can be drawn from these data are generally consistent with the conclusions we have drawn from the results of the temperature calculations. Only the larger value of the $\mathrm{C} 1$ 
calculation error looks a little strange in comparison with the A2 calculation. In general, we can state the sufficient accuracy of the analytical solution for the estimated calculations.

\section{Conclusions}

1. The solution of the unsteady thermal conductivity problem for a bilayer cylinder with an arbitrary initial temperature distribution and a linearly varying ambient temperature is constructed. This solution has a simple form, is expressed in terms of elementary functions and can be easily implemented using any computer package. An algorithm for using the above solution to find temperature fields in the case of piecewise constant change in the heat transfer coefficient has been proposed. The solution of the thermal conductivity problem is supplemented by the solution of the corresponding thermoelasticity problem for a bilayer cylinder, as well as by the solution of the problem of a bilayer cylinder under internal pressure. This whole set of analytical relations makes it possible to obtain temperature and stress distributions in bilayer cylindrical vessels and pipelines subjected to arbitrary axisymmetric force and temperature loading.

2. The accuracy of the analytical solution is sufficient for practical calculations. If the time intervals at which the heat transfer coefficient is assumed to be constant are chosen correctly, deviations of both temperatures and stresses do not exceed 3\%. In this case, the main contribution to this deviation is made by averaging of temperature-dependent material properties. With a non-axisymmetric distribution of the temperature load, the proposed solution can serve to estimate the stress level and sort the scenarios under consideration according to the degree of danger.

\section{References}

[1] Carslaw H. S., Jaeger J. C. Conduction of Heat in Solids. Oxford: Oxford University, 1959. 510 p.

[2] Ozisik M. N. Heat conduction in the cylindrical coordinate system. In Boundary Value Problems of Heat Conduction, International Textbook Company, Scranton, Chapter 3, 1968, pp. 125-193.

[3] Tu T.W., Lee S.Y. Analytical Solution of Heat Conduction for Hollow Cylinders with TimeDependent Boundary Condition and Time-Dependent Heat Transfer Coefficient. J. Appl. Math., Vol. 2015, 2015, Art. ID 203404, 9 p.

[4] Lee S.Y., Huang C.C., Tu T.W. Transient Heat Conduction in Nonuniform Hollow Cylinders with Time-Dependent Boundary Condition at One Surface. IJAME, Vol. 9 (7), 2015, pp. 1317-1322.

[5] Kadenko I., Sakhno N., Kutzenko O., Kharytonov O. An Analytical Approach to the Generation of the Operational Limit Curves with NDE-based Crack Postulation. Proc. 8h Intl. Conf. on NDE in Relation to Structural Integrity for Nuclear and Pressurised Components, Berlin, 2010, pp. 234242.

[6] Kutsenko O., Kadenko I., Kharytonov O., Sakhno N. An analytical basis for the generation of NPP emergency operation limiting pressure-temperature curves. Mathematical Modeling and Computing, Vol. 3 (1), 2016, pp. 79-89.

[7] Pressurized Thermal Shock in Nuclear Power Plants: Good Practices for Assessment (IAEA TECDOC 1627). Vienna: IAEA, 2010, 217 p.

[8] Doetsch G. Introduction to the Theory and Application of the Laplace Transformation. - New York: Springer-Verlag, 1974, $326 \mathrm{p}$.

[9] Timoshenko S.P., Goodier J.N. Theory of Elasticity. New-York: McGraw-Hill, 1970, 591 p.

[10]Правила и положения по проектированию и анализу напряжений для компонентов и трубопроводов ядерной установки (Rules and Regulations for Design and Stress Analysis for Components and Piping of Nuclear Installation) (PNAEG-G7-002-86). Moscow: Energoatomizdat, 1989, 525 p. (In Russian)

[11] Kutsenko O., Kadenko I., Pitoiset X., Kharytonov O.M., Sakhno N., Kravchenko I. Effect of neutron irradiation hardening of the base metal on the results of WWER-1000 reactor pressure vessel residual lifetime assessment. Int. J. Press. Vessel. Pip., Vol. 171, 2019, pp. 173-183.

[12] Dhondt G. The Finite Element Method for Three-Dimensional Thermomechanical Applications. Hoboken: Wiley, 2004, 340 p. 